\title{
Treewidth of graphs with balanced separations
}

\author{
Zdeněk Dvořák* $\quad$ Sergey Norin ${ }^{\dagger}$
}

\begin{abstract}
We prove that if every subgraph of a graph $G$ has a balanced separation of order at most $a$ then $G$ has treewidth at most $15 a$. This establishes a linear dependence between the treewidth and the separation number.
\end{abstract}

\section{Introduction and results}

Treewidth of a graph is an important graph parameter introduced by Robertson and Seymour [8], which expresses how "tree-like" a given graph is. The relation between the treewidth and other graph parameters, e.g. the maximum order of a tangle [10] and the size of a largest grid-minor [9, 11, 3], has been explored in a number of papers; see [7] for a recent survey. The goal of this paper is to establish a linear dependence between treewidth and another parameter, the separation number, which we now define.

A separation of a graph $G$ is a pair $(A, B)$ of subsets of $V(G)$ such that $A \cup B=V(G)$ and no edge of $G$ has one end in $A \backslash B$ and the other in $B \backslash A$. The order of the separation $(A, B)$ is $|A \cap B|$. For an assignment $w: V(G) \rightarrow \mathbb{R}_{0}^{+}$of non-negative weights to vertices of $G$ and a set $X \subseteq V(G)$, we define $w(X)=\sum_{v \in X} w(v)$. We say that a separation $(A, B)$ of a graph $G$ is $w$-balanced if $w(A \backslash B) \leq \frac{2}{3} w(V(G))$ and $w(B \backslash A) \leq \frac{2}{3} w(V(G))$. A separation $(A, B)$ of a graph $G$ on $n$ vertices is balanced if $|A \backslash B| \leq 2 n / 3$ and $|B \backslash A| \leq 2 n / 3$, i.e., it is $w$-balanced for the function $w$ assigning weight 1

\footnotetext{
${ }^{*}$ Charles University, Prague, Czech Republic. E-mail: rakdver@iuuk.mff.cuni.cz. Supported by the Center of Excellence - Inst. for Theor. Comp. Sci., Prague (project P202/12/G061 of Czech Science Foundation).

${ }^{\dagger}$ Department of Mathematics and Statistics, McGill University. Email: snorin@math.mcgill.ca
} 
to each vertex of $G$. The separation number $\operatorname{sn}(G)$ of a graph $G$ is the smallest integer $s$ such that every subgraph of $G$ has a balanced separation of order at most $s$.

Let $\operatorname{tw}(G)$ denote the treewidth 1 of the graph $G$. The relation between the separation number and the treewidth has been explored starting with Robertson and Seymour [8]. They proved that if $G$ has treewidth at most $t$, then for every assignment $w$ of non-negative weights to vertices of $G$, there exists a $w$-balanced separation of $G$ of order at most $t+1$. Conversely, they also showed that if $G$ has a $w$-balanced separation of order at most $s$ for every $\{0,1\}$-valued weight assignment $w$, then the treewidth of $G$ is less than $4 s$.

Since $\operatorname{tw}\left(G^{\prime}\right) \leq \operatorname{tw}(G)$ for every subgraph $G^{\prime}$ of $G$, it follows that $\operatorname{sn}(G) \leq$ tw $(G)+1$ for every graph $G$. In this paper, we study whether a weak converse to this inequality holds. Note that this is not obvious: the fact that each subgraph has a balanced separation of small order does not straightforwardly imply that the whole graph has a $w$-balanced separation of small order for every $\{0,1\}$-valued weight assignment $w$.

It is easy to show that $\operatorname{tw}(G) \leq 1+\operatorname{sn}(G) \log (|V(G)|)$, see e.g. [1]. Fox [6] stated without proof that $\operatorname{sn}(G)$ and $\operatorname{tw}(G)$ are tied, that is $\operatorname{tw}(G) \leq f(\operatorname{sn}(G))$ for some function $f$. Finally, Böttcher et al. 2] investigated the relation between the separation number and the treewidth for graphs with bounded maximum degree. They have shown that for fixed $\Delta$ and a subgraph-closed class of graphs with maximum degree at most $\Delta$, the treewidth is sublinear in the number of vertices if and only if the separation number is.

The following is our main result.

Theorem 1. The treewidth of any graph $G$ is at most $15 \operatorname{sn}(G)$.

Note that Theorem 1 implies the aforementioned result of Böttcher et al. without the restriction on the maximum degree. We give the necessary definitions and prove the preliminary results in Section 2, The proof of Theorem 1 is completed in Section 3 .

\section{Brambles and flows}

In this section we introduce the tools and auxiliary results necessary for the proof of Theorem 1 .

\footnotetext{
${ }^{1}$ We will not need the definition of treewidth in this paper. Instead, we use an equivalent parameter, the maximum order of a bramble, which is defined in Section 2 ,
} 


\subsection{Brambles}

Let $G$ be a graph. A set $\mathcal{B}$ of non-empty subsets of $V(G)$ is a bramble if the induced subgraph $G[S]$ is connected for all $S \in \mathcal{B}$ and the induced subgraph of $G\left[S_{1} \cup S_{2}\right]$ is connected for all $S_{1}, S_{2} \in \mathcal{B}$. A hitting set for a bramble $\mathcal{B}$ is a set that intersects all elements of $\mathcal{B}$. The order of the bramble is the smallest size of its hitting set. The relation between the brambles and the treewidth of a graph is captured in the following result of Seymour and Thomas.

Theorem 2 (Seymour and Thomas [12]). A graph $G$ contains a bramble of order $k$ if and only if $G$ has treewidth at least $k-1$.

A flap assignment of order $k$ in a graph $G$ is a function $\beta$ that maps each set $W \subseteq V(G)$ of size less than $k$ to the vertex set of a (non-empty) connected component of $G-W$. A haven of order $k$ is a flap assignment of order $k$ such that for all sets $W, W^{\prime} \subseteq V(G)$ of sizes less than $k$, the induced subgraph $G\left[\beta(W) \cup \beta\left(W^{\prime}\right)\right]$ is connected. Clearly, if $\beta$ is a haven of order $k$ in $G$, then $\{\beta(W): W \subseteq V(G),|W|<k\}$ forms a bramble in $G$ of order at least $k$.

Conversely, consider a bramble $\mathcal{B}$ in $G$ of order $k$. If a set $W \subseteq V(G)$ has size smaller than $k$, then $W$ is not a hitting set for $\mathcal{B}$, and thus there exists a set $S \in \mathcal{B}$ disjoint from $W$. Since $G[S]$ is connected, there exists a component $C$ of $G-W$ containing $S$. Furthermore, since $G\left[S_{1} \cup S_{2}\right]$ is connected for all $S_{1}, S_{2} \in \mathcal{B}$, all elements of $\mathcal{B}$ disjoint from $W$ are subsets of this unique component $C$. Hence, setting $\beta(W)$ to be the vertex set of such component $C$ for each $W \subseteq V(G)$ of size less than $k$ gives a haven $\beta$ of order $k$, which we call the haven defined by the bramble $\mathcal{B}$.

Let us note a basic property of havens.

Observation 3. Let $\beta$ be a haven of order $k$ in a graph $G$, let $W_{1}$ and $W_{2}$ be sets of vertices of $G$ of size less than $k$, and let $(A, B)$ be a separation of $G$ of order less than $k$. If $W_{1} \subseteq W_{2}$, then $\beta\left(W_{2}\right) \subseteq \beta\left(W_{1}\right)$. Also, if $\beta\left(W_{1}\right) \subseteq A \backslash B$, then $\beta(A \cap B) \subseteq A \backslash B$.

We need the following result enabling us to find a small(er) set in the image of a haven.

Lemma 4. Let $a, r \geq 0$ be integers and let $G$ be a graph with separation number at most $a$. Let $Y$ be a subset of vertices of $G$, and let $\beta$ be a haven in $G$ of order greater than $|Y|+$ ar. Then there exists a set $Y^{\prime}$ of vertices of $G$ such that $\left|Y^{\prime}\right| \leq|Y|+$ ar and $\left|\beta\left(Y^{\prime}\right)\right| \leq(2 / 3)^{r}|\beta(Y)|$. 
Proof. We prove the claim by induction on $r$; for $r=0$, the claim clearly holds, hence assume that $r \geq 1$. Let $G_{1}$ be the subgraph of $G$ induced by $\beta(Y)$. Since $\operatorname{sn}(G) \leq a$, there exists a balanced separation $(A, B)$ of $G_{1}$ of order at most $a$. Let $Y_{1}=Y \cup(A \cap B)$. By Observation 3, we have $\beta\left(Y_{1}\right) \subseteq \beta(Y)=V\left(G_{1}\right)$. Since $\beta\left(Y_{1}\right)$ is disjoint from $A \cap B$ and induces a connected subgraph, we can by symmetry assume that $\beta\left(Y_{1}\right) \subseteq A \backslash B$. Since the separation $(A, B)$ is balanced, we have $\left|\beta\left(Y_{1}\right)\right| \leq|A \backslash B| \leq \frac{2}{3}|\beta(Y)|$. Since $\left|Y_{1}\right| \leq|Y|+a$, the claim of the lemma follows by the induction hypothesis applied to $Y_{1}$ and $r-1$.

\subsection{Flows and clouds}

Let $\vec{G}$ be a directed graph and let $g: V(\vec{G}) \rightarrow \mathbb{R}_{0}^{+}$be a function specifying the amount of flow entering its vertices. The value $g(V(\vec{G})):=\sum_{v \in V(\vec{G})} g(v)$ is called the total supply. Let $W=\left\{v_{1}, v_{2}, \ldots, v_{k}\right\} \subseteq V(\vec{G})$ be a set of sinks in $\vec{G}$, where a sink is a vertex with no outgoing edges. A flow towards $W$ in $\vec{G}$ with supply $g$ is a function $f: E(\vec{G}) \rightarrow \mathbb{R}_{0}^{+}$satisfying the flow conservation equation

$$
g(v)+\sum_{e=u v \in E(\vec{G})} f(e)=\sum_{e=v z \in E(\vec{G})} f(e)
$$

for every $v \in V(\vec{G}) \backslash W$. For a fixed flow $f$ and a vertex $v \in V(\vec{G})$, define $\operatorname{in}(v)$ to be the sum of the flow values on the edges into $v$. The congestion at $v$ is defined as in $(v)+g(v)$. The congestion of a flow $f$ is the maximum congestion over all the vertices of the graph. Note that the total supply equals the sum of congestions of vertices of $W$. Let $\|f\|=\sum_{e \in E(\vec{G})} f(e)$ and let the support $\operatorname{supp}(f)$ of $f$ be the set of edges of $\vec{G}$ with non-zero value of the flow.

Let $G$ be an undirected graph and let $W$ be a subset of $V(G)$. The graph $\vec{G}_{W}$ is obtained from $G$ by replacing each edge with a pair of oppositely directed edges and deleting the edges directed away from the vertices of $W$. A $W$-cloud in $G$ is a flow in $\vec{G}_{W}$ towards $W$ such that the supply at each vertex is at most 1 . The following result is an easy corollary of the max-flow min-cut theorem (applied to the graph obtained from $\vec{G}_{W}$ by splitting each vertex into an input and an output part connected by an edge of capacity $\alpha$, adding a source vertex with edges of capacity 1 going into all input parts of vertices of $\vec{G}_{W}$, and having the capacity of all the other edges infinite). 
Observation 5. Let $G$ be an undirected graph and let $W$ be a subset of $V(G)$, and let $\alpha, s>0$ be real numbers. There exists a $W$-cloud in $G$ with congestion at most $\alpha$ and total supply greater than $s$ if and only if $G$ contains no separation $(C, D)$ with $W \subseteq C$ and $|C \backslash D|+\alpha|C \cap D| \leq s$.

Let $G$ be an undirected graph and let $W$ be a subset of $V(G)$. For a $W$-cloud $f$ with supply $g$, a vertex $v \in V(G)$ is saturated if $g(v)=1$, and $v$ is hungry if $v$ is not saturated, but has non-zero congestion or belongs to $W$. Let sat $(f)$ denote the set of saturated vertices of $G$. Let $\vec{G}_{W} \downarrow f$ denote the subgraph of $\vec{G}_{W}$ whose vertices are all hungry and saturated vertices of $\vec{G}$ and with edge set equal to the support of $f$, and let $G_{W} \downarrow f$ denote the underlying undirected graph of $\vec{G}_{W} \downarrow f$. We say that a $W$-cloud $f$ is $\alpha$-tame if the congestion of $f$ is at most $\alpha$ and additionally,

(i) the hungry vertices have no in-neighbors in $\vec{G}_{W} \downarrow f$,

(ii) each saturated vertex has at most one hungry in-neighbor in $\vec{G}_{W} \downarrow f$, and

(iii) if $\alpha \leq 1$, then $\vec{G}_{W} \downarrow f$ is the edgeless graph with vertex set $W$.

Lemma 6. Let $G$ be an undirected graph, let $W$ be a subset of $V(G)$, and let $\alpha, s>0$ be real numbers. If there exists any $W$-cloud in $G$ with congestion at most $\alpha$ and total supply exactly s, then there also exists an $\alpha$-tame $W$-cloud with total supply exactly s.

Proof. Let $f$ be a $W$-cloud in $G$ with congestion at most $\alpha$ and total supply exactly $s$ such that among all such $W$-clouds, $\|f\|$ is minimum, and subject to that, $|\operatorname{supp}(f)|-|\operatorname{sat}(f)|$ is minimum. Note that such a $W$-cloud exists by the compactness of the space of all $W$-clouds with given total supply and upper bound on congestion.

If $\alpha \leq 1$, then let $f^{\prime}$ be the flow with the empty support and with supply at each vertex of $W$ equal to the congestion of $f$ at $W$. Since $\|f\| \leq\left\|f^{\prime}\right\|=0$, we have $f=f^{\prime}$ and $\vec{G}_{W} \downarrow f$ is the edgeless graph with vertex set $W$. Clearly, $f$ is $\alpha$-tame.

Hence, assume that $\alpha>1$, and in particular the condition (iii) of $\alpha$ tameness is trivially satisfied. The graph $\vec{G}_{W} \downarrow f$ is acyclic, since if $C$ were a directed cycle in $\vec{G}_{W} \downarrow f$, we could decrease the values of the flow on the edges of $C$ by the same positive amount and obtain a $W$-cloud $f^{\prime}$ with congestion at most $\alpha$ and total supply $s$ such that $\left\|f^{\prime}\right\|<\|f\|$. 
Let $g$ be the supply of $f$. Suppose first that there exists an edge $u v \in$ $E\left(\vec{G}_{W} \downarrow f\right)$ such that $v$ is hungry. Let $P$ be a maximal directed path in $\vec{G}_{W} \downarrow f$ ending with the edge $u v$. Let $\delta_{1}>0$ be the minimum of the flow values on the edges of $P$. Since $\vec{G}_{W} \downarrow f$ is acyclic, the starting vertex $x$ of $P$ has no incoming edges, and thus $g(x) \geq \delta_{1}$. Let $\delta=\min \left(\delta_{1}, 1-g(v)\right)$. Let $f^{\prime}$ be obtained from $f$ by decreasing the flow by $\delta$ on the edges of $P$, decreasing the supply at $x$ by $\delta$ and increasing the supply at $v$ by $\delta$. Clearly, $f^{\prime}$ is a $W$-cloud with the same total supply and at most as large congestion as $f$, and $\left\|f^{\prime}\right\|<\|f\|$, which is a contradiction. Hence, hungry vertices have no in-neighbors in $\vec{G}_{W} \downarrow f$, and $f$ satisfies the condition (i) of $\alpha$-tameness.

Suppose now that there exist distinct edges $v_{1} z, v_{2} z \in E\left(\vec{G}_{W} \downarrow f\right)$ such that $v_{1}$ and $v_{2}$ are hungry. By the previous paragraph, $v_{1}$ has no incoming edges, and thus $g\left(v_{1}\right) \geq f\left(v_{1} z\right)$. Let $\delta=\min \left(f\left(v_{1} z\right), 1-g\left(v_{2}\right)\right)$. Let $f^{\prime}$ be the flow obtained by decreasing the flow on $v_{1} z$ by $\delta$, increasing the flow on $v_{2} z$ by $\delta$, decreasing the supply at $v_{1}$ by $\delta$ and increasing the supply at $v_{2}$ by $\delta$. Only the congestion of $v_{2}$ increases, and since $v_{2}$ has no in-neighbors by the previous paragraph, the congestion of $f^{\prime}$ at $v_{2}$ is at most its supply at $v_{2}$, which is at most $1 \leq \alpha$. Hence, $f^{\prime}$ is a $W$-cloud with the same total supply as $f$ and congestion at most $\alpha$, and $\left\|f^{\prime}\right\|=\|f\|$. Furthermore, either $\operatorname{supp}\left(f^{\prime}\right)=\operatorname{supp}(f) \backslash\left\{v_{1} z\right\}$, or $\operatorname{sat}\left(f^{\prime}\right)=\operatorname{sat}(f) \cup\left\{v_{2}\right\}$, and thus $\left|\operatorname{supp}\left(f^{\prime}\right)\right|-\left|\operatorname{sat}\left(f^{\prime}\right)\right|<|\operatorname{supp}(f)|-|\operatorname{sat}(f)|$, which is a contradiction. We conclude that each vertex has at most one hungry in-neighbor in $\vec{G}_{W} \downarrow f$, and $f$ satisfies the condition (ii) of $\alpha$-tameness.

Therefore, $f$ is $\alpha$-tame.

We now use clouds to extract a special subgraph of a hypothetical counterexample to Theorem 1 ,

Lemma 7. Let $G$ be a graph and let $a=\operatorname{sn}(G)$. If $a \geq 1$ and $G$ has treewidth at least 15 a, then $G$ contains a subgraph $F$ with a separation $(X, Z)$ of order 14 a such that the following conditions hold. Let $W=X \cap Z, s=14|Z \backslash W|$ and $\alpha=\frac{s}{7 a}$.

(a) The subgraph $F[X]$ contains an $\alpha$-tame $W$-cloud $f$ with total supply greater than s, such that each vertex of $F[X]$ is hungry or saturated.

(b) There exists a balanced separation $(A, B)$ of $F$ such that

$$
|A \cap(W \cup B) \cap X| \leq a .
$$


Proof. By Theorem 2, $G$ contains a bramble $\mathcal{B}$ of order at least $15 a+1$. Let $\beta$ denote the haven of order at least $15 a+1$ defined by $\mathcal{B}$. Let $W$ be a subset of vertices of $G$ of size at most $14 a$ chosen so that $|\beta(W)|$ is minimum, and subject to that $|W|$ is minimum. Clearly, $|W|=14 a$, as otherwise for any $w \in \beta(W)$, we have $\beta(W \cup\{w\}) \subsetneq \beta(W)$, contradicting the minimality of $|\beta(W)|$. Similarly, the minimality of $|W|$ implies that every vertex of $W$ has a neighbor in $\beta(W)$. Let $X^{\prime}=V(G) \backslash \beta(W)$ and $Z=W \cup \beta(W)$. Let $s=14|Z \backslash W|=14|\beta(W)|$ and $\alpha=\frac{s}{7 a}$.

Suppose first that $G\left[X^{\prime}\right]$ has a separation $(C, D)$ such that $W \subseteq C$ and $|C \backslash D|+\alpha|C \cap D| \leq s$, and thus the order of $(C, D)$ is at most $s / \alpha=7 a$ and $|C \backslash D| \leq s=14|\beta(W)|$. Let $C^{\prime}=C \cup \beta(W)$ and $Y=C^{\prime} \cap D=C \cap D$, and note that $\left(C^{\prime}, D\right)$ is a separation of $G$. Since $\beta(W) \subseteq C^{\prime} \backslash D$, Observation 3 implies $\beta(Y) \subseteq C^{\prime} \backslash D$. Consequently, $|\beta(Y)| \leq\left|C^{\prime} \backslash D\right|=|\beta(W)|+|C \backslash D| \leq$ $15|\beta(W)|$. Applying Lemma 4 with $r=7$, we conclude that there exists a set $Y^{\prime} \subseteq V(G)$ of size at most $|Y|+r a \leq 14 a$ such that $\left|\beta\left(Y^{\prime}\right)\right| \leq(2 / 3)^{7}|\beta(Y)| \leq$ $15(2 / 3)^{7}|\beta(W)|<|\beta(W)|$, which contradicts the choice of $W$.

Therefore, $G\left[X^{\prime}\right]$ has no such separation, and thus by Observation 5, $G\left[X^{\prime}\right]$ contains a $W$-cloud $f$ of congestion at most $\alpha$ and total supply $s_{f}>s$. Furthermore, by Lemma 6, we can assume that $f$ is $\alpha$-tame. Let $X$ be the set of hungry and saturated vertices of $G\left[X^{\prime}\right]$ (so $X=V\left(G\left[X^{\prime}\right]_{W} \downarrow f\right)$ ) and let $F=\left(G\left[X^{\prime}\right]_{W} \downarrow f\right) \cup G[Z]$. Clearly, $(X, Z)$ is a separation of $F, X \cap Z=W$ has size $14 a$, and $f$ is an $\alpha$-tame $W$-cloud in $F[X]$ satisfying (a).

Since $\operatorname{sn}(G)=a$ and $F$ is a subgraph of $G$, there exists a balanced separation $(A, B)$ of $F$ of order at most $a$. Let $\left(A^{\prime}, B^{\prime}\right)=(A \cap Z, B \cap Z)$ be the separation of $G[Z]$ corresponding to $(A, B)$. If $B^{\prime} \backslash A^{\prime}$ contains all vertices of $\beta(W)$, then $W \subseteq B^{\prime}$, since each vertex of $W$ has a neighbor in $\beta(W)$. Consequently $W \subseteq B$ and $A \cap(W \cup B) \cap X \subseteq A \cap B$, implying (b). Hence, we can assume that $\beta(W) \nsubseteq B^{\prime} \backslash A^{\prime}$, and symmetrically (swapping the labels of $A$ and $B$ ), we have $\beta(W) \nsubseteq A^{\prime} \backslash B^{\prime}$.

Let $W^{\prime}=W \cup\left(A^{\prime} \cap B^{\prime}\right)$. We have $\left|W^{\prime}\right| \leq 15 a$, and thus $\beta\left(W^{\prime}\right)$ is defined. Since $W \subseteq W^{\prime}$, we have $\beta\left(W^{\prime}\right) \subseteq \beta(W)$, and by symmetry (swapping the labels of $A$ and $B$ if necessary), we can assume that $\beta\left(W^{\prime}\right) \subseteq B^{\prime} \backslash\left(A^{\prime} \cup W\right) \subsetneq$ $\beta(W)$. Let $W^{\prime \prime}=W^{\prime} \cap B^{\prime}$. No vertex of $W \backslash B^{\prime}$ has a neighbor in $B^{\prime} \backslash A^{\prime}$, and thus $\beta\left(W^{\prime \prime}\right)=\beta\left(W^{\prime}\right)$. Consequently, the minimality of $|\beta(W)|$ implies that $\left|W^{\prime \prime}\right|>14 a=|W|$, and thus $|A \cap(W \cup B) \cap X|=|A \cap B|+|A \cap W|-$ $|A \cap B \cap Z|=|A \cap B|+|W|-\left|W^{\prime \prime}\right|<|A \cap B| \leq a$. Therefore, (b) holds.

We are now ready to prove Theorem 1 by showing that the conditions (a) 
and (b) are contradictory.

\section{Proof of Theorem 1}

Let $a=\operatorname{sn}(G)$. We assume without loss of generality that $a \geq 1$. Suppose for a contradiction that $G$ has treewidth at least 15a. By Lemma 7, $G$ contains a subgraph $F$ with a separation $(X, Z)$ of order $14 a$ satisfying (with $W=X \cap Z, s=14|Z \backslash W|$ and $\alpha=\frac{s}{7 a}$ ) conditions (a) and (b). Let $(A, B)$ be a balanced separation of $F$ such that

$$
|A \cap(W \cup B) \cap X| \leq a,
$$

which exists by (b).

By (a), $F[X]$ contains an $\alpha$-tame $W$-cloud $f$ with total supply greater than $s$, such that each vertex of $F[X]$ is hungry or saturated, and thus $X=$ $V\left(F[X]_{W} \downarrow f\right)$. If $\alpha<1 / 2$, then $X=W$ by the condition (iii) of $\alpha$-tameness. Consequently, $V(F)=Z$. Furthermore, $|Z \backslash W|=s / 14=\alpha a / 2<a / 4$. By (11), we have $|A \cap W| \leq a$, and thus

$$
\frac{|B \backslash A|}{|V(F)|} \geq \frac{|W|-|A \cap W|}{|W|+|Z \backslash W|}>\frac{13 a}{(14+1 / 4) a}>2 / 3 .
$$

This contradicts the fact that $(A, B)$ is a balanced separation of $F$, and thus $\alpha \geq 1 / 2$.

Let $g$ be the supply of the $W$-cloud $f$. Let $a_{0}=|A \cap(W \cup B) \cap X|$ and let $a_{1} \leq a_{0}$ be the number of hungry vertices in $A \cap(W \cup B) \cap X$. Note that by the condition (i) of $\alpha$-tameness, no flow enters the hungry vertices of $A \cap(W \cup B) \cap X$ through incident edges. Consider a saturated vertex $v \in A \cap X$. The flow $g(v)=1$ originating at $v$ must reach $W$, and thus it must pass through (or end in) a saturated vertex of $A \cap(W \cup B) \cap X$. Since all vertices have congestion at most $\alpha$, it follows that the number of saturated vertices of $A \cap X$ is at most $\alpha\left(a_{0}-a_{1}\right)$. By the condition (ii) of $\alpha$-tameness, each saturated vertex of $A \cap X$ has at most one hungry in-neighbor, and

each hungry vertex $x$ of $A \cap X$ either belongs to $A \cap(W \cup B) \cap X$ or has a saturated out-neighbor in $A \cap X$. Since $a_{0} \leq a$ by (1), we conclude that

$$
|A| \leq|A \cap X|+|Z \backslash W| \leq 2 \alpha\left(a_{0}-a_{1}\right)+a_{1}+|Z \backslash W| \leq 2 \alpha a+|Z \backslash W|=\frac{5}{14} s .
$$


On the other hand, the total supply of $f$ is greater than $s$ and each vertex has supply at most 1 , giving the lower bound greater than $s$ on the size of $X$. Hence, we have

$$
|V(F)|=|X|+|Z \backslash W|>s+|Z \backslash W|=\frac{15}{14} s .
$$

Consequently,

$$
\frac{|B \backslash A|}{|V(F)|}=1-\frac{|A|}{|V(F)|}>1-\frac{5 s / 14}{15 s / 14}=2 / 3 .
$$

This contradiction to the fact that $(A, B)$ is a balanced separation of $F$ finishes the proof.

\section{Acknowledgments}

We would like to thank the anonymous referee whose suggestions helped us to significantly improve the multiplicative constant in Theorem 1. An earlier version of the paper [5] used results of Chen, Kleinberg, Lovász, Rajaraman, Sundaram, and Vetta [4] regarding confluent flows; while we were able to avoid their usage eventually, they inspired the method we use.

\section{References}

[1] H. L. Bodlaender, A partial k-arboretum of graphs with bounded treewidth, Theoretical Computer Science, 209 (1998), pp. 1-45.

[2] J. Böttcher, K. P. Pruessmann, A. Taraz, and A. Würfl, Bandwidth, expansion, treewidth, separators and universality for bounded-degree graphs, European J. Combin., 31 (2010), pp. 1217-1227.

[3] C. Chekuri and J. Chuzhoy, Polynomial bounds for the grid-minor theorem, J. ACM, 63 (2016), pp. 40:1-40:65.

[4] J. Chen, R. D. Kleinberg, L. Lovász, R. Rajaraman, R. SunDARAM, AND A. VETTA, (Almost) tight bounds and existence theorems for single-commodity confluent flows, J. ACM, 54 (2007), pp. 16:1-16:32. 
[5] Z. Dvoř́́K AND S. NoRIN, Treewidth of graphs with balanced separations. http://arxiv.org/abs/1408.3869v1.

[6] J. Fox, Constructing dense graphs with sublinear Hadwiger number. J. Combin. Theory Ser. B, to appear.

[7] D. Harvey And D. Wood, Parameters tied to treewidth, Journal of Graph Theory, 84 (2017), pp. 364-385.

[8] N. Robertson And P. D. Seymour, Graph minors. II. Algorithmic aspects of tree-width, Journal of Algorithms, 7 (1986), pp. 309-322.

[9] N. Robertson And P. D. Seymour, Graph Minors. V. Excluding a planar graph, J. Combin. Theory, Ser. B, 41 (1986), pp. 92-114.

[10] N. Robertson And P. D. Seymour, Graph Minors. X. Obstructions to tree-decomposition, J. Combin. Theory, Ser. B, 52 (1991), pp. 153190.

[11] N. Robertson, P. D. Seymour, and R. Thomas, Quickly excluding a planar graph, J. Combin. Theory, Ser. B, 62 (1994), pp. 323-348.

[12] P. D. Seymour And R. Thomas, Graph searching and a min-max theorem for tree-width, Journal of Combinatorial Theory, Series B, 58 (1993), pp. 22-33. 